\title{
Bio-protective effect of a root-nodulating Rhizobium etli strain in common bean (Phaseolus vulgaris) against Meloidogyne incognita and Radopholus similis in an in vitro autotrophic tripartite culture system
}

\author{
Lieselot VAn der Veken ${ }^{1}$, Pa Pa Win $^{2}$, Preeti Seeboruth ${ }^{3}$, Ma. Teodora N. Cabas An ${ }^{4, *}$, \\ Rony SWEnNEN ${ }^{5,6}$, Annemie Elsen ${ }^{7,8}$ and Dirk De WAEle ${ }^{5,9}$ \\ ${ }^{1}$ Pro Terra Agro, Bijlokstraat 144, B-3020 Herent, Belgium \\ ${ }^{2}$ Livelihoods and Food Security Fund (LIFT, UNOPS), Mayangone Township, Yangon, Myanmar \\ ${ }^{3}$ Faculty of Agriculture, University of Mauritius, Reduit 80837, Mauritius \\ ${ }^{4}$ Department of Biological Sciences, College of Science and Mathematics, University of Southern Mindanao, \\ 9407 Kabacan, Cotabato, Philippines \\ ${ }^{5}$ Laboratory of Tropical Crop Improvement, Department of Biosystems, Faculty of Bioscience Engineering, \\ University of Leuven (KULeuven), W. De Croylaan 42, B-3001 Leuven, Belgium \\ ${ }^{6}$ International Institute of Tropical Agriculture (IITA), Arusha, Tanzania \\ ${ }^{7}$ Soil Service of Belgium, W. De Croylaan 48, B-3001 Heverlee, Belgium \\ ${ }^{8}$ Department of Biology, Faculty of Sciences, Ghent University, K.L. Ledeganckstraat 35, B-9000 Gent, Belgium \\ ${ }^{9}$ Unit for Environmental Sciences and Management, North-West University, Private Bag X6001, Potchefstroom 2520,
}

South Africa

Received: 7 September 2020; revised: 16 October 2020 Accepted for publication: 17 October 2020

\begin{abstract}
Summary - The bio-protective effect of a root-nodulating strain (CNPAF 512) of the nitrogen-fixing rhizobium, Rhizobium etli, against both a sedentary (Meloidogyne incognita) and a migratory (Radopholus similis) endoparasitic nematode in common bean (Phaseolus vulgaris) was examined using an in vitro autotrophic tripartite culture system. Two in vitro assays were carried out with each of the nematode species. Each assay consisted of two treatments: the plants were either inoculated with the rhizobial strain or remained noninoculated (control plants). To examine the effect of either pre- or simultaneous inoculation of the rhizobial strain on the reproduction of $M$. incognita and R. similis, one assay was carried out in which the nematodes were inoculated 3 weeks after rhizobial inoculation while another assay was carried out in which the nematodes were inoculated simultaneously with the rihizobial strain. Both preinoculation and simultaneous inoculation with $R$. etli CNPAF 512 significantly suppressed the reproduction of both $M$. incognita and R. similis.
\end{abstract}

Keywords - autotrophic model system, biocontrol, burrowing nematode, nodulation, plant growth-promoting rhizobacteria, root-knot nematode.

Common bean (Phaseolus vulgaris L.) is the most widely cultivated food legume throughout the world, providing a primary source of dietary protein, fibre, starch and minerals affordable by the poor (Broughton et al., 2003; Martinez-Romero, 2003). It is considered an 'allaround' crop that can be grown either as a rotation crop, intercrop or green manure crop in a wide range of agro- ecological systems (Broughton et al., 2003). As with most other species of the legume family (Fabaceae), common bean can form a symbiosis with nitrogen-fixing soil bacteria (collectively known as rhizobia) within a specialised structure, the root nodule (van Rhijn \& Vanderleyden, 1995; Martinez-Romero, 2003; Soares et al., 2016). Rhizobia can take up gaseous dinitrogen $\left(\mathrm{N}_{2}\right)$ from the air

\footnotetext{
* Corresponding author, e-mail: mtncabasan@usm.edu.ph
} 
and 'fix' it into ammonia that can subsequently be assimilated into amino acids by the bacterium or the plant. In return, the plant provides the rhizobia with a carbon source in the form of dicarboxylic acids (van Rhijn \& Vanderleyden, 1995). Although common bean has good potential for $\mathrm{N}_{2}$ fixation, it was reported to have the lowest $\mathrm{N}_{2}$ fixation rate among the most widely grown grain legumes (Martinez-Romero, 2003). Most of the rhizobia originally isolated from tropical legumes from humid or sub-humid tropical forests from the Amazon and the south-east of Brazil (the centre of origin of common bean) were able to induce nodules or nodular structures on common bean, but only a minority of these nodules were also nitrogen-fixing, indicating a high specificity for effective $\mathrm{N}_{2}$ fixation (Michiels et al., 1998). A nitrogenfixing plant produces leghaemoglobin, a protein related to human haemoglobin, for providing oxygen to the nodules, giving functional nodules a pink colour (Soares et al., 2016). Rhizobium etli is the predominant species found in the centres of origin of common bean in Central and South America (Martínez-Romero, 2003).

Everywhere throughout the tropics and subtropics common bean is attacked by a diversity of plant-parasitic nematodes, among which the root-knot nematodes ( $M e$ loidogyne spp.) are the most important. The most common root-knot nematode species infecting common bean are Meloidogyne incognita, M. javanica and M. arenaria (Sikora et al., 2018). Root-knot nematodes are sedentary endoparasites. Also, root-lesion nematodes (Pratylenchus spp.) have been reported to infect common bean causing extensive root necrosis and yield loss (Sikora et al., 2018). The most typical symptom caused by root-knot nematodes are galls (knots), and by root-lesion nematodes root lesions, purplish-black necrotic areas that usually extend throughout the cortex (Jones et al., 2013).

Rhizobia belong to the group of plant growth-promoting rhizobacteria (PGPR) that are able to enhance the tolerance of their hosts to both abiotic and biotic stresses (Verma et al., 2019). Research on the effect of root nodulation by rhizobia on plant-parasitic nematodes and vice versa has mainly dealt with the interaction between soybean and Heterodera glycines, the soybean cyst nematode, and between a variety of leguminous crops and the most common root-knot nematode species, especially $M$. incognita. In most instances, root nodulation by rhizobia suppressed root galling and reproduction of $M$. incognita and improved plant growth (Huang, 1987; Fazal et al., 1992; Khan et al., 2018), whilst infection by M. incognita reduced either the number of nodules, their functionality or induced premature senescence of the nodules (see, for example, Baldwin et al., 1979; Huang, 1987; Khan et al., 2002, 2018; Neeraj \& Singh, 2019). Similarly, infection by Pratylenchus penetrans affected the number, weight and functionality of nodules (Elhady et al., 2020). Information on the effect of root nodulation by rhizobia on migratory endoparasitic nematodes is scarce. Recently, Van der Veken et al. (unpubl.) found that simultaneous inoculation of soybean with $R$. etli CNPAF 512 and Radopholus similis, a root-lesion nematode, significantly suppressed reproduction of this migratory endoparasite.

The objective of our study was to evaluate the bioprotective effect of the root-nodulating strain $R$. etli CNPAF 512 against both a sedentary (M. incognita) and a migratory ( $R$. similis) endoparasitic nematode in common bean. The experiment was carried out using an in vitro autotrophic tripartite culture system.

\section{Materials and methods}

\section{PlANT MATERIAL}

Common bean 'BAT 477' seeds were obtained from the Centre of Microbial and Plant Genetics (CMPG), University of Leuven, Belgium. This cultivar was selected because its roots develop well in vitro and it is susceptible to both $M$. incognita and $R$. similis. The seeds were placed in a Miracloth pouch in a sterilised Erlenmeyer flask and surface-sterilised by submersion in $97 \%$ ethanol for $1 \mathrm{~min}$ and $15 \% \mathrm{NaOCl}$ for $12 \mathrm{~min}$. The Erlenmeyer flask was shaken constantly to assure close contact between the seeds and the disinfectants. The seeds were then rinsed ten times with sterile demineralised water $\left(\mathrm{dH}_{2} \mathrm{O}\right)$ for $1 \mathrm{~min}$ and soaked in $\mathrm{dH}_{2} \mathrm{O}$ for $1 \mathrm{~h}$. Subsequently, the seeds were removed from the Miracloth pouch with sterilised tweezers and 4-5 sterilised seeds transferred to sterilised 9-cm diam. Petri dishes containing a sterilised water agar medium for germination. The Petri dishes were sealed with parafilm and placed in an incubator at $25^{\circ} \mathrm{C}$ with a 12:12 light:dark photoperiod.

\section{BACTERIAL INOCULUM}

The $R$. etli CNPAF 512 strain was also obtained from CMPG. This bacterial strain was selected because of its good compatibility with common bean 'BAT 477' (Michiels et al., 1998). The bacterial strain was first cultured for $2-3$ days at $28^{\circ} \mathrm{C}$ on a solid tryptone-yeast (TY) medium containing $5 \mathrm{~g}$ tryptone, $3 \mathrm{~g}$ yeast extract 

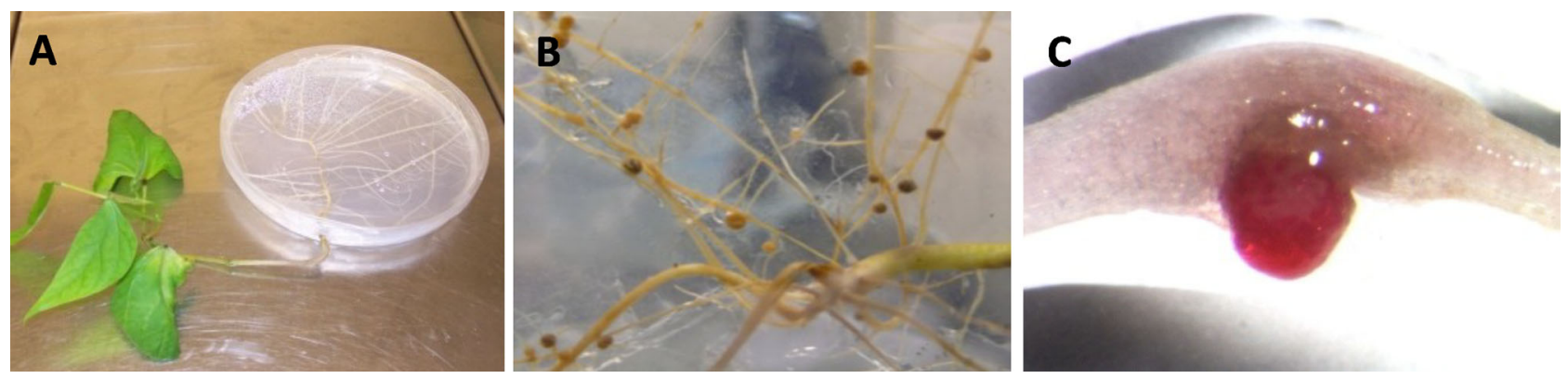

Fig. 1. A: In vitro autotrophic tripartite culture system of common bean; B: Nodulation of roots of common bean inoculated with Rhizobium etli CNPAF 512; C: Egg mass of Meloidogyne incognita after Phloxine B staining.

with $15 \mathrm{~g}$ plant agar $\mathrm{l}^{-1}$ (Bittinger \& Handelsman, 2000) followed by 1 day on a liquid TY medium at $28^{\circ} \mathrm{C}$ and $200 \mathrm{rpm}$. Then $1 \%$ of a $10 \mathrm{mM} \mathrm{CaCl} \cdot \mathrm{H}_{2} \mathrm{O}$ solution was added to promote growth of the bacterial cell wall. The optical density (OD) of the bacterial suspension was determined by measuring its absorbance at $600 \mathrm{~nm}$ wavelength using a spectrophotometer. At the time of inoculation, the bacterial suspension was diluted in a $10 \mathrm{mM} \mathrm{MgSO} 4$ solution to obtain a bacterial inoculum containing about $10^{6}$ colony forming units (CFU) $\mathrm{ml}^{-1}$.

\section{NEMATODE INOCULUM}

The $M$. incognita population used in the trials was originally collected from a banana field in Malaysia and maintained on susceptible tomato 'Marmande' plants in soil in pots in a glasshouse. The inoculum consisted of infective second-stage juveniles (J2) that were extracted from infected tomato roots by a modified Baermann technique. Under sterile conditions in a laminar flow chamber and using a stereomicroscope, nematode egg masses protruding from infected tomato roots were removed with a scalpel and transferred to a 20-mm-diam. microsieve (70- $\mu \mathrm{m}$ diam. mesh), which was placed in the centre of a watch glass containing sterile $\mathrm{dH}_{2} \mathrm{O}$. The watch glass was then placed in a Petri dish which was subsequently sealed with parafilm and placed in an incubator at $25^{\circ} \mathrm{C}$ in the dark. Six days later, the majority of J2 had hatched and were collected by rinsing the watch glass with sterile $\mathrm{dH}_{2} \mathrm{O}$.

The $R$. similis population used in the trials was originally collected from a banana field in Uganda and maintained on a monoxenic alfalfa callus culture grown on a modified White's medium in Petri dishes at $27^{\circ} \mathrm{C}$ in the dark (Elsen et al., 2001). The inoculum consisted of approximately 60 females, which were collected under ster- ile conditions in a laminar flow chamber by rinsing the callus medium with sterile $\mathrm{dH}_{2} \mathrm{O}$.

\section{EXPERIMENTAL SET-UP}

To examine the bio-protective effect of root nodulation on $M$. incognita and R. similis on common bean, an in vitro autotrophic tripartite culture system was developed based on a autotrophic culture system for the in vitro mycorrhization of potato plantlets (Voets et al., 2005; Fig. 1). This system consisted of sterilised $14.5-\mathrm{cm}$ diam. closed and sealed Petri dishes containing a $\mathrm{pH}$-adjusted (pH 6.2) sugar- and nitrogen-free Snoeck agar medium (Snoeck et al., 2003). The root system of a sterilised 5-day-old common bean 'BAT 477' seedling was gently pushed through an opening (made in the side of each of the Petri dishes with a heated cork borer) and into the medium until the tap rootlet reached the centre of the Petri dish. After insertion of the root system, the opening in the side of the Petri dish was sealed with silicon grease. In this way, an even growth of the seedlings' roots over the medium inside the Petri dishes could be obtained, while the shoots were growing outside the Petri dishes to allow normal photosynthesis.

Two in vitro assays were carried out with each of the nematode species. Each assay consisted of two treatments: the plants were either inoculated with the rhizobial strain $\left(\mathrm{RHIZ}^{+}\right)$or remained non-inoculated (RHIZ ${ }^{-}$; control plants). To examine the effect of either pre- or simultaneous inoculation of the rhizobial strain on the reproduction of $M$. incognita and $R$. similis, one assay was carried out in which the nematodes were inoculated 3 weeks after rhizobial inoculation while another assay was carried out in which the nematodes were inoculated simultaneously with the rihizobial strain. Petri dishes were arranged in a randomised block design. Each treatment was replicated 
either six or eight times. The simultaneous inoculation of R. etli CNPAF 512 and M. incognita assay was repeated.

Rhizobial inoculation $\left(\mathrm{RHIZ}^{+}\right.$) was done by pipetting $1 \mathrm{ml}$ of the $R$. etli CNPAF 512 inoculum suspension (containing $\pm 10^{6} \mathrm{CFU}$ ) over the 5-day-old tap rootlets, allowing any excess of the suspension to drip onto the medium. Control plants $\left(\mathrm{RHIZ}^{-}\right)$only received $1 \mathrm{ml}$ of the $10 \mathrm{mM} \mathrm{MgSO}$ solution. For the pre-inoculation treatment, the nematodes were inoculated 3 weeks after inoculation with $R$. etli CNPAF 512, when nodules had already formed. A $1 \mathrm{ml}$ aqueous suspension containing either $c a 60 \mathrm{~J} 2$ of $M$. incognita or ca 60 females of $R$. similis was pipetted on the medium near the root tips of the plantlets (both $\mathrm{RHIZ}^{+}$and $\mathrm{RHIZ}^{-}$plants). For the simultaneous inoculation treatment, the nematodes were inoculated as described above at the same time as the rhizobial inoculation (both $\mathrm{RHIZ}^{+}$and $\mathrm{RHIZ}^{-}$ plants). After inoculation, the Petri dishes were sealed with parafilm and the opening in the side of the Petri dish around the stems of the seedling sealed with silicon grease. Petri dishes were stacked randomly with the shoots facing the same direction, wrapped with aluminum foil to mimic underground conditions for optimal root growth and placed in a growth chamber at $25^{\circ} \mathrm{C}, 70 \% \mathrm{RH}$ and a 12:12 light:dark photoperiod.

\section{ASSESSMENT OF ROOT GROWTH, RHIZOBIAL COLONISATION AND NEMATODE REPRODUCTION}

Eight weeks after nematode inoculation, the Petri dishes were opened, the root systems gently removed from the medium and rinsed with tap water. The length of the tap root was measured. Rhizobial colonisation was assessed by counting the number of nodules that had developed on the whole root system using a stereomicroscope (Fig. 1B) while the inside colour of the nodules was observed for the presence of leghaemoglobin. For M. incognita, egg-laying females (ELF) were stained red (Fig. 1C) by soaking the rinsed root systems in a Phloxine B solution $\left(0.15 \mathrm{~g} \mathrm{l}^{-1}\right)$ for 15 min (Holbrook et al., 1983), and ELF and egg masses counted using a stereomicroscope. The $\mathrm{J} 2$ and males were extracted from the medium using a Baermann tray (Hooper et al., 2005) and counted after $48 \mathrm{~h}$ of extraction in two $2 \mathrm{ml}$ subsamples of a $50 \mathrm{ml}$ suspension using a light microscope. For $R$. similis, root necrosis was first scored on a 0 to 7 scale: $0=$ absence of lesions; $1=$ sporadic lesions and traces of infections in $<5 \%$ of the roots; $2=$ larger coalesced lesions in 5 to up to $10 \%$ of the roots; $3=$ necrotic root parts present in 10 to up to $25 \%$ of the roots; $4=25 \%$ of roots necrotic and lesions visible in the other roots; $5=$ between 25 and up to $50 \%$ of roots necrotic and lesions visible in the other roots; $6=$ between 50 and up to $75 \%$ of roots necrotic and lesions visible in the other roots; $7=>75 \%$ of roots necrotic. Subsequently, juveniles and adults were extracted from the roots by the maceration-sieving technique (Hooper et al., 2005) and from the medium as described for $M$. incognita above. Number of eggs, juveniles, females and males were counted in two $2 \mathrm{ml}$ subsamples of a $50 \mathrm{ml}$ suspension using a light microscope.

\section{STATISTICAL ANALYSIS}

Data were analysed separately for each assay. Nematode data were $\log (\mathrm{x}+1)$ transformed prior to statistical analysis. One-way ANOVA and post-hoc Tukey equal HSD test were performed using Statistica 7.1 software after verifying the ANOVA assumptions (Anonymous, 2007). The categorical root necrosis data were analysed using the Kruskal-Wallis test.

\section{Results}

In all assays, the common bean plantlets developed a good root system (Fig. 1A, B) with root lengths averaging 20-25 cm (assays with $M$. incognita). Rhizobial inoculation was also successful in all assays. On the plants of the pre-inoculation assays infected with $M$. incognita, on average 43 and 27 nodules were counted in trials 1 and 2, respectively, while on the plants of the simultaneous inoculation assay with $M$. incognita, on average 87 nodules were counted (Fig. 2A). On the plants of the pre-inoculation and simultaneous inoculation assays infected with $R$. similis, on average 45 and 71 nodules, respectively, were counted (Fig. 2B). Active nitrogen fixation by the rhizobia was observed visually by the presence of nodules with a pink centre, indicating the presence of leghaemoglobin.

Pre-inoculation with $R$. etli CNPAF 512 significantly $(P \leqslant 0.05)$ reduced the number of $M$. incognita ELF, egg masses in the roots (by 40 and 50\%) and number of $\mathrm{J} 2$ in the medium (by 57 and 59\%) but not the number of males in both trials 1 and 2, respectively (Table 1). A similar result was obtained in the simultaneous inoculation assay, but in this assay the number of males was also significantly $(P \leqslant 0.05)$ reduced (by $\pm 40 \%)$. In the simultaneous inoculation assay, a much higher number of males of M. incognita was observed compared with the preinoculation assay in both non-inoculated plants and plants 

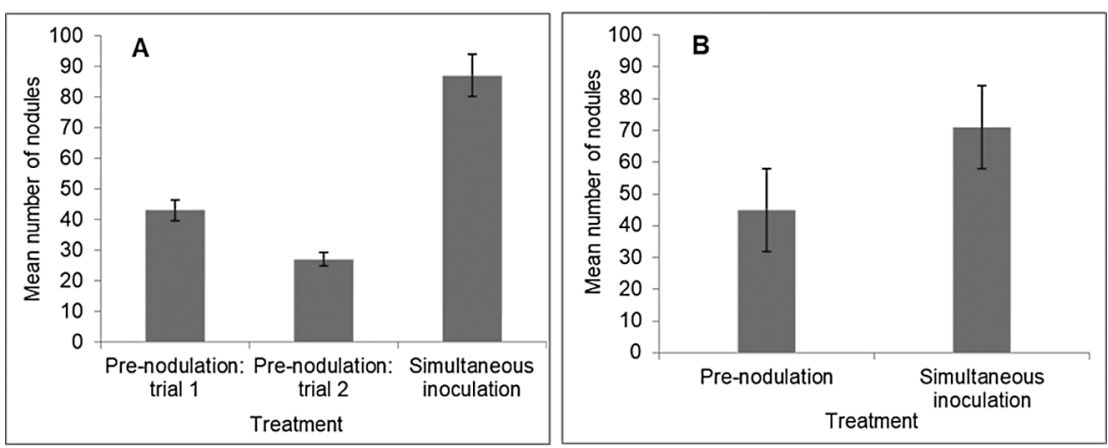

Fig. 2. Mean number of nodules per root system that had developed on common bean 8 weeks after inoculation with Meloidogyne incognita (A) or Radopholus similis (B). Nematode inoculation was done either 3 weeks after inoculation with Rhizobium etli CNPAF 512 (pre-nodulation) or simultaneously with inoculation of the rhizobial strain. Bars indicate standard errors of the means.

Table 1. Effect of pre-inoculation with Rhizobium etli followed 3 weeks later by inoculation with $c a$ second-stage juveniles (J2) of Meloidogyne incognita (pre-inoculation assay) and simultaneous inoculation with both $R$. etli and ca $60 \mathrm{~J} 2$ of M. incognita (simultaneous inoculation assay) on the reproduction of $M$. incognita and root length of common bean plants, grown in an in vitro autotrophic tripartite culturing system, at 8 weeks after nematode inoculation.

\begin{tabular}{|c|c|c|c|c|c|c|}
\hline \multirow[t]{2}{*}{ Treatment } & \multirow[t]{2}{*}{$\mathrm{n}$} & \multicolumn{4}{|c|}{ Number } & \multirow[t]{2}{*}{ Root length $(\mathrm{cm})$} \\
\hline & & $\mathrm{J} 2$ & $\begin{array}{c}\text { Egg-laying } \\
\text { females (ELF) }\end{array}$ & Egg masses & Males & \\
\hline \multicolumn{7}{|c|}{ Pre-inoculation with $R$. etli followed by inoculation with $M$. incognita } \\
\hline \multicolumn{7}{|l|}{ Trial 1} \\
\hline $\mathrm{RHIZ}^{-}$ & 8 & 228 & 10 & 8 & 8 & 20.6 \\
\hline \multirow[t]{2}{*}{$\mathrm{RHIZ}^{+}$} & 8 & 98 & 6 & 3 & 14 & 23.3 \\
\hline & & ** & $* * *$ & $* * *$ & ns & ns \\
\hline \multicolumn{7}{|l|}{ Trial 2} \\
\hline RHIZ $^{-}$ & 8 & 355 & 10 & 8 & 16 & 20.0 \\
\hline \multirow[t]{2}{*}{$\mathrm{RHIZ}^{+}$} & 8 & 147 & 5 & 3 & 23 & 22.7 \\
\hline & & $* * *$ & $* * *$ & $* * *$ & $\mathrm{~ns}$ & ns \\
\hline \multicolumn{7}{|c|}{ Simultaneous inoculation of $R$. etli and $M$. incognita } \\
\hline $\mathrm{RHIZ}^{-}$ & 6 & 216 & 9 & 8 & 206 & 25.0 \\
\hline \multirow[t]{2}{*}{$\mathrm{RHIZ}^{+}$} & 6 & 73 & 5 & 3 & 125 & 23.1 \\
\hline & & $* * *$ & $* * *$ & $* * *$ & $*$ & ns \\
\hline
\end{tabular}

$\mathrm{n}=$ number of replicates. $\mathrm{RHIZ}^{-}$: plants inoculated with Meloidogyne incognita only; $\mathrm{RHIZ}^{+}$: plants inoculated with Rhizobium etli and Meloidogyne incognita.

${ }^{*},{ }^{* *}$ and ${ }^{* * *}$ indicate significant differences at $P \leqslant 0.05,0.01$ and 0.001 , respectively, according to Tukey's equal HSD test; ns: not significant.

inoculated with $R$. etli CNPAF 512. No significant differences in root length were observed. However, greener and more abundant leaves were observed in plants inoculated with $R$. etli CNPAF 512.

Pre-inoculation with $R$. etli CNPAF 512 significantly $(P \leqslant 0.05)$ reduced the number of juveniles and females of $R$. similis but not the number of eggs and males; root necrosis was also significantly $(P \leqslant 0.05)$ reduced
(Table 2). In the simultaneous inoculation assay, the number of eggs, juveniles, females and males of $R$. similis, and root necrosis were significantly $(P \leqslant 0.05)$ reduced in plants inoculated with $R$. etli compared with noninoculated plants. In the simultaneous inoculation assay, much lower numbers of eggs, juveniles and females, but not males, of $R$. similis were observed compared with the pre-inoculation assay in both non-inoculated plants and 
Table 2. Effect of pre-inoculation with Rhizobium etli followed 3 weeks later by inoculation with ca 60 females of Radopholus similis (pre-inoculation assay) and simultaneous inoculation with both $R$. etli and $c a 60$ females of $R$. similis (simultaneous inoculation assay) on the reproduction of $R$. similis and root necrosis of common bean plants, grown in an in vitro autotrophic tripartite culturing system, at 8 weeks after nematode inoculation.

\begin{tabular}{|c|c|c|c|c|c|c|}
\hline \multirow[t]{2}{*}{ Treatment } & \multirow[t]{2}{*}{$\mathrm{n}$} & \multicolumn{4}{|c|}{ Number } & \multirow[t]{2}{*}{ Root necrosi } \\
\hline & & Eggs & Juveniles & Females & Males & \\
\hline \multicolumn{7}{|c|}{ Pre-inoculation with $R$. etli followed by inoculation with $R$. similis } \\
\hline $\mathrm{RHIZ}^{-}$ & 8 & 206 & 2517 & 585 & 125 & 3 \\
\hline \multirow[t]{2}{*}{$\mathrm{RHIZ}^{+}$} & 8 & 1037 & 1275 & 294 & 161 & 2 \\
\hline & & ns & ** & $* *$ & ns & ** \\
\hline \multicolumn{7}{|c|}{ Simultaneous inoculation of $R$. etli and $R$. similis } \\
\hline $\mathrm{RHIZ}^{-}$ & 6 & 44 & 129 & 52 & 306 & 5 \\
\hline \multirow[t]{2}{*}{$\mathrm{RHIZ}^{+}$} & 6 & 17 & 39 & 23 & 175 & 3 \\
\hline & & $* *$ & $* * *$ & $* *$ & $*$ & $* *$ \\
\hline
\end{tabular}

$\mathrm{n}=$ number of replicates. RHIZ ${ }^{-}$: plants inoculated with Radopholus similis only; $\mathrm{RHIZ}^{+}$: plants inoculated with Rhizobium etli and Radopholus similis.

Root necrosis on a 0 to 7 scale: $0=$ absence of lesions; $1=$ sporadic lesions and traces of infections in $<5 \%$ of the roots; $2=$ larger coalesced lesions in 5 to up to $10 \%$ of the roots; $3=$ necrotic root parts present in 10 to up to $25 \%$ of the roots; $4=25 \%$ of roots necrotic and lesions visible in the other roots; $5=$ between 25 and up to $50 \%$ of roots necrotic and lesions visible in the other roots; $6=$ between 50 and up to $75 \%$ of roots necrotic and lesions visible in the other roots; $7=>75 \%$ of roots necrotic.

${ }^{*},{ }^{* *}$ and ${ }^{* * *}$ indicate significant differences at $P \leqslant 0.05,0.01$ and 0.001 , respectively, according to Tukey's equal HSD test or the Kruskal-Wallis test (root necrosis); ns: not significant.

plants inoculated with $R$. etli CNPAF 512. Nodulation of plants was significantly $(P \leqslant 0.05)$ lower in the preinoculation assays with $M$. incognita (both trials) and $R$. similis compared with the simultaneous inoculation assays.

\section{Discussion}

The common bean plantlets developed a good root system, rhizobial inoculation resulted in functional nodules, and the infective developmental stages of $M$. incognita and $R$. similis infected the plantlets and developed and reproduced successfully inside the root systems. All these observations validated the in vitro autotrophic tripartite culture system as a highly controlled, reproducible and easy to use model system to study plant-rhizobiaendoparasitic nematode interactions. Autotrophic plant growth, in the absence of sugar in the medium, results in normal photosynthetic-active plant tissues, hormonal balance and physiological source-sink relationships, which were considered major limitations of the use of excised organ cultures to study in vitro the interactions between plants and either beneficial organisms or pathogens, because they may alter the plant's physiology (Fortin et al.,
2002; Voets et al., 2005). Also, this model system presumably allowed the plant to release root exudates in the agar, which specifically induced the synthesis of nodulation factors in rhizobia that initiated the nodulation process (Peters et al., 1986; Pandya et al., 1999) and attracted the infective developmental stages of the plant-parasitic nematodes to the roots (Perry \& Curtis, 2013). Finally, it allows monitoring throughout the duration of the experiment plant growth, nodulation and, in the case of rootknot nematodes, also root galling and the emergence of egg masses from the roots.

Both pre-inoculation with $R$. etli CNPAF 512 and simultaneous inoculation significantly suppressed reproduction of the sedentary endoparasitic nematode $M$. incognita on common bean. This result confirms previous observations of the bio-protective suppression of $M$. incognita following either pre- or simultaneous inoculation of leguminous crops (chickpea, mungbean, lentil, pigeonpea) with rhizobia (Fazal et al., 1992; Khan et al., 2018). Both pre-inoculation with $R$. etli CNPAF 512 and simultaneous inoculation also significantly suppressed the reproduction of the migratory endoparasitic nematode $R$. similis on common bean. This result confirmed (Van der Veken et al., data unpubl.) that simultaneous inoculation 
of soybean with $R$. etli CNPAF 512 and $R$. similis significantly suppressed nematode reproduction. For both assays with $M$. incognita and $R$. similis, no differences were observed between the bioprotective effect of pre-inoculation with $R$. etli CNPAF 512 and simultaneous inoculation. Nodulation significantly suppressed the number of $R$. similis males in the simultaneous inoculation assay but not in the pre-inoculation assay. For the other developmental stages (eggs, juveniles and females) this was not the case. Males are often rare in root-knot nematode populations but in some species such as $M$. incognita more J2 developed into males under adverse conditions (Jones et al., 2013). It is possible that an unknown stress factor during the simultaneous inoculation assay resulted in the development of more males, and that simultaneous inoculation of $R$. etli CNPAF 512 and $\mathrm{J} 2$ of $M$. incognita also resulted in suppression of the number of males that developed.

In contrast with the mechanism involved in the nematode-suppressive effects of arbuscular mycorrhizal fungi (AMF) on plant-parasitic nematodes (Schouteden et al., 2015), the mechanisms involved in the bio-protective effects of rhizobia against plant-parasitic nematodes are much less studied. Competition for nutrients and space between $R$. etli CNPAF 512 and M. incognita, aggravated in the confined space in which the root systems of common bean had to develop in our in vitro assays, may have played a role in the observed nematode-suppressive effect against this root-knot nematode species. Competition for nutrients is considered a potential mechanism for suppression of plant-parasitic nematodes by endophytic bacteria (Hallmann et al., 1997). In potato, R. etli G12, a rhizosphere coloniser with endophytic potential, preferentially colonised the root tips and emerging lateral roots, which were also the preferential penetration sites for the infective J2 of root-knot nematodes (Jones et al., 2013), and approximately $20 \%$ of the galls induced by $M$. incognita (Hallmann et al., 2001). Based on the large numbers of $R$. etli G12 that were often observed in the gall tissues, Hallmann et al. (2001) suggested that leaked nutrients became available to the endophytic bacteria. Root-knot nematodeinduced giant cells inside the galls act as a metabolic sink and contain 4-6 times more glucose and free amino acids than actively growing root tip cells (Huang, 1985). These nutrients were transported from the surrounding tissues into the giant cells and it is possible that endophytic bacteria intercept and take up these nutrients. Fewer nutrients would then become available for the developing females of $M$. incognita, interfering with their development and reproduction. In our assays with $M$. incognita, it was obvious, based on the high numbers of nodules, that $R$. etli CNPAF 512 was highly successful in colonising the roots of the common bean plantlets. Visual observation of the plantlets showed that the nodules and galls induced by $M$. incognita were often situated closely together. This may have limited the penetration sites available for the infective $\mathrm{J} 2$ and the sites inside the roots where the $\mathrm{J} 2$ could establish a feeding site. Competition for nutrients between rhizobia and migratory endoparasitic nematodes, such as $R$. similis, has not been reported so far but it cannot be excluded that the mechanisms described above to explain the bio-protective effect of $R$. etli CNPAF 512 against $M$. incognita also apply for $R$. similis. Migratory endoparasites do not establish feeding sites but feed upon the cells of the cortex when migrating through the root system causing leakage of the nutrients inside these cells. However, no $R$. similis was recovered from the nodules so we do not expect competition for nutrients to have played a significant role for the observed nematodesuppressive effect against $R$. similis. Another mechanism that may have played a role in the observed nematodesuppressive effect against both $M$. incognita and $R$. similis was non-pathogenic rhizobacteria-mediated induced systemic resistance (ISR), a systemic resistance in plants that is phenotypically similar to pathogen-induced systemic acquired resistance (SAR; van Loon et al., 1998).

The observation that nodulation of the common bean root systems was significantly lower in the pre-inoculation assays with $M$. incognita (both trials) compared with the simultaneous inoculation assays confirmed numerous previous reports that $M$. incognita infection (and also $R$. similis as demonstrated by our study) may also affect root nodulation by rhizobia (see, for example, Huang, 1987; Sharma \& Tiagi, 1990; Khan et al., 2002, 2018). The observation that in both assays with $M$. incognita and $R$. similis, a significantly higher number of nodules had developed on the root systems of the simultaneous inoculation assay compared with the pre-inoculation assay suggests that the simultaneous inoculation of $R$. etli CNPAF 512 and the nematodes may have facilitated, in one way or the other, root penetration and colonisation by the rhizobia, for instance, by rhizobia adhering to the cuticle of the nematodes when these penetrated and migrated through the roots. By contrast, on pea, a greater reduction in the number of nodules was observed when $M$. incognita was inoculated simultaneously with a Rhizobium strain compared with when the Rhizobium strain was inoculated 2 weeks before $M$. incognita (a reduction of $30 \mathrm{vs} 12 \%$, respectively; Sharma \& Tiagi, 1990). However, on lentil, no 
difference in the number of nodules was observed when a Rhizobium strain was inoculated either together with or 10 days before M. incognita (Fazal et al., 1992). On soybean, higher numbers of nodules formed on plants infested with $P$. penetrans compared with the non-infested plants after inoculation with Bradyrhizobium japonicum; however, when nematodes invaded roots with established nodules the number of nodules was not affected (Elhady et al., 2020).

In both the pre-inoculation and simultaneous assays with both $M$. incognita and $R$. similis, no significant increase in root growth (tap root length) was observed in both trials between the $\mathrm{RHIZ}^{-}$and $\mathrm{RHIZ}^{+}$treatments. However, in the RHIZ- treatment infected with M. incognita, nematode infection was more severe (more J2, ELF and egg masses) compared with the $\mathrm{RHIZ}^{+}$treatment, resulting in a lower shoot weight compared with the RHIZ ${ }^{+}$ treatment (data not shown). Thus, $R$. etli CNPAF 512 limited these adverse effects of $M$. incognita on plant growth by suppressing the nematode's reproduction. Most probably, the growth-promoting effect of $R$. etli CNPAF 512 on root growth (tap root length) was limited due to the confined space in which the root systems had to develop in our in vitro assays.

\section{Acknowledgements}

This research was made possible thanks to the University of Leuven (KU Leuven; Special Research Fund) Ph.D. scholarship to L. Van der Veken, a Flemish Interuniversity Council (VLIR-UOS) Ph.D. scholarship to P.P. Win and a Research Foundation-Flanders (FWOVlaanderen) postdoctoral fellowship to A. Elsen. We also like to thank Anke Massart, all the seed suppliers, and Maarten Fauvart and Roseline Remans of the Centre of Microbial and Plant Genetics (CMPG) of the University of Leuven (KU Leuven) for providing us with the common bean seeds and the bacterial strain.

\section{References}

Anonymous (2007). Statistica Package 7.1. Tulsa, OK, USA, Statsoft.

Baldwin, J.G., Barker, K.R. \& Nelson, L.A. (1979). Effects of Meloidogyne incognita on nitrogen fixation in soybean. Journal of Nematology 11, 156-161.

Bittinger, M.A. \& Handelsman, J. (2000). Identification of genes in the RosR regulon of Rhizobium etli. Journal of
Bacteriology 6, 1706-1713. DOI: 10.1128/jb.182.6.17061713.2000

Broughton, W.J., Hernández, G., Blair, M., Beebe, S., Gepts, P. \& Vanderleyden, J. (2003). Beans (Phaseolus spp.) - model food legumes. Plant and Soil 252, 55-128. DOI: 10.1023/A: 1024146710611

Elhady, A., Hallman, J. \& Heuer, H. (2020). Symbiosis of soybean with nitrogen fixing bacteria affected by root lesion nematodes in a density-dependent manner. Scientific Reports 10, 1619. DOI: 10.1038/s41598-020-58546-x

Elsen, A., Lens, K., Nguyet, D.T.M., Broos, S., Stoffelen, R. \& De Waele, D. (2001). Aseptic culture systems of Radopholus similis for in vitro assays on Musa spp. and Arabidopsis thaliana. Journal of Nematology 33, 147-151.

Fazal, M., Siddiqui, Z.A. \& Imran, M. (1992). Effect of pre-, post- and simultaneous inoculations of Rhizobium, Rotylenchulus reniformis and Meloidogyne incognita on lentil. Nematologia Mediterranea 20, 159-161.

Fortin, J.A., Bécard, G., Declerck, S., Dalpé, Y., St-Arnaud, M., Coughlan, A.P. \& Piché, Y. (2002). Arbuscular mycorrhiza on root-organ cultures. Canadian Journal of Botany 80, 1-20. DOI: $10.1139 / \mathrm{b} 01-139$

Hallmann, J., Quadt-Hallmann, A., Mahaffee, W.F. \& Kloepper, J.W. (1997). Bacterial endophytes in agricultural crops. Canadian Journal of Microbiology 43, 895-914. DOI: 10. 1139/m97-131

Hallmann, J., Quadt-Hallmann, A., Miller, W.G., Sikora, R.A. \& Lindow, S.E. (2001). Endophytic colonization of plants by the biocontrol agent Rhizobium etli G12 in relation to Meloidogyne incognita infection. Phytopathology 91, 415422. DOI: 10.1094/PHYTO.2001.91.4.415

Holbrook, C.C., Knauft, D.A. \& Dickson, D.W. (1983). A technique for screening peanut resistance to Meloidogyne incognita. Plant Disease 67, 957-958.

Hooper, D.J., Hallman, J. \& Subbotin, S. (2005). Methods for extraction, processing and detection of plant and soil nematodes. In: Luc, M., Sikora, R.A. \& Bridge, J. (Eds). Plant parasitic nematodes in subtropical and tropical agriculture, 2nd edition. Wallingford, UK, CAB International, pp. 53-86.

Huang, C.S. (1985). Formation, anatomy and physiology of giant cells induced by root-knot nematodes. In: Sasser, J.N. \& Carter, C.C. (Eds). An advanced treatise on Meloidogyne. Vol. 1. Biology and control. Raleigh, NC, USA, North Carolina State University Graphics, pp. 154-164.

Huang, J.S. (1987). Interaction of nematodes with rhizobia. In: Veech, J.A. \& Dickson, D.W. (Eds). Vistas on nematology: a commemoration of the 25th anniversary of the society of nematologists. Hyattsville, Maryland, USA, Society of Nematologists Inc., pp. 301-306.

Jones, J.T., Haegeman, A., Danchin, E.G.J., Gaur, H.S., Helder, J., Jones, M.G.K., Kikuchi, T., Manzanilla-López, R., Palomares-Rius, J.E., Wesemael, W.M.L. et al. (2013). Top 10 plant-parasitic nematodes in molecular plant pathology. 
Molecular Plant Pathology 14, 946-961. DOI: 10.1111/mpp. 12057

Khan, M.R., Kounsar, K. \& Hamid, A. (2002). Effect of certain rhizobacteria and antagonistic fungi on root-nodulation and root-knot nematode disease of green gram. Nematologia Mediterranea 30, 85-89.

Khan, M.R., Mohiddin, F.A. \& Ahamad, F. (2018). Inoculant rhizobia suppressed root-knot disease and enhanced plant productivity and nutrient uptake of some field-grown food legumes. Acta Agriculturae Scandinavica, Section B - Soil \& Plant Science 68, 166-174. DOI: 10.1080/09064710.2017. 1374448

Martínez-Romero, E. (2003). Diversity of Rhizobium-Phaseolus vulgaris symbiosis: overview and perspectives. Plant and Soil 252, 11-23. DOI: 10.1023/A:1024199013926

Michiels, J., Dombrecht, B., Vermeiren, N., Xi, C., Luyten, E. \& Vanderleyden, J. (1998). Phaseolus vulgaris is a non-selective host for nodulation. FEMS Microbiology Ecology 26, 193205. DOI: 10.1111/j.1574-6941.1998.tb00505.x

Neeraj \& Singh, G. (2019). Quantifying the interaction between root-knot nematode (Meloidogyne incognita) and rhizobium on Moong (Vigna radiata). International Journal of Current Microbiology and Applied Sciences 8, 2180-2184. DOI: 10. 20546/ijcmas.2019.804.256

Pandya, S., Iyer, P., Gaitonde, V., Parekh, T. \& Desai, A. (1999). Chemotaxis of Rhizobium sp.S2 towards Cajanus cajan root exudate and its major components. Current Microbiology 38, 205-209. DOI: 10.1007/PL00006788

Perry, R.N. \& Curtis, R.H.C. (2013). Behaviour and sensory perception. In: Perry, R.N. \& Moens, M. (Eds). Plant nematology, 2nd edition. Wallingford, UK, CAB International, pp. 246-273.

Peters, N.K., Frost, J.W. \& Long, S.R. (1986). A plant flavone, luteolin, induces expression of Rhizobium meliloti nodulation genes. Science 233, 977-980. DOI: 10.1126/science.3738520

Schouteden, N., De Waele, D., Panis, B. \& Vos, C.M. (2015). Arbuscular mycorrhizal fungi for the biocontrol of plantparasitic nematodes: a review of the mechanisms involved.
Frontiers in Microbiology 6, 1280. DOI: 10.3389/fmicb.2015. 01280

Sharma, R.K. \& Tiagi, B. (1990). Effect of Meloidogyne incognita on nodulation and symbiotic nitrogen fixation in pea. Nematologia Mediterranea 18, 15-17.

Sikora, R.A., Claudius-Cole, B. \& Sikora, E.J. (2018). Nematode parasites of food legumes. In: Sikora, R.A., Coyne, D., Hallmann, J. \& Timper, P. (Eds). Plant parasitic nematodes in subtropical and tropical agriculture, 3rd edition. Wallingford, UK, CAB International, pp. 290-345.

Snoeck, C., Verreth, C., Hernandez-Lucas, I., Martínez-Romero, E. \& Vanderleyden, J. (2003). Identification of a third sulfate activation system in Sinorhizobium sp. strain BR816: the CysDN sulfate activation complex. Applied and Environmental Microbiology 69, 2006-2014. DOI: 10.1128/AEM.69.4. 2006-2014.2003

Soares, R., Trejo, J., Veloso, M.M. \& Videira e Castro, I. (2016). [Biological nitrogen fixation by Phaseolus vulgaris.] Revista de Ciências Agrárias 39, 526-537. DOI: 10.19084/ RCA16104

van Loon, L.C., Bakker, P.A.H.M. \& Pieterse, M.J. (1998). Systemic resistance induced by rhizosphere bacteria. Annual Review of Phytopathology 36, 453-483. DOI: 10.1146/annurev. phyto.36.1.453

van Rhijn, P. \& Vanderleyden, J. (1995). The Rhizobium-plant symbiosis. Microbiological Reviews 59, 124-142.

Verma, P.P., Shelake, R.M., Das, S., Sharma, P. \& Kim, J.Y. (2019). Plant growth-promoting rhizobacteria (PGPR) and fungi (PGPF): potential biological control agents of diseases and pests. In: Singh, D.P., Gupta, V.K. \& Prabh, R. (Eds). Microbial interventions in agriculture and development. Vol. 1: research trends, priorities and prospects. Cham, Switzerland, Springer International Publishing, pp. 281-311.

Voets, L., Dupré de Boulois, H., Renard, L., Strullu, D.G. \& Declerck, S. (2005). Development of an autotrophic culture system for the in vitro mycorrhization of potato plantlets. FEMS Microbiology Letters 248, 111-118. DOI: 10.1016/j. femsle.2005.05.025 